\title{
Effects of Wrist-extension Orthosis on Shoulder and Scapular Muscle Activities during Simulated Assembly Tasks
}

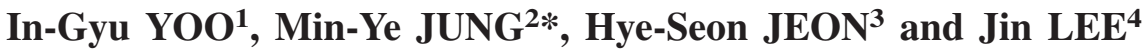 \\ ${ }^{1}$ Department of Occupational Therapy, The Graduate School, Yonsei University, 234 Maeji-ri, Heungup-myun, \\ Wonju, Kangwon-do 220-710, Republic of Korea \\ ${ }^{2}$ Department of Occupational Therapy, College of Health Science, Yonsei University, 234 Maeji-ri, Heungup- \\ myun, Wonju, Kangwon-do 220-710, Republic of Korea \\ ${ }^{3}$ Department of Physical Therapy, College of Health Science, Yonsei University, 234 Maeji-ri, Heungup-myun, \\ Wonju, Kangwon-do 220-710, Republic of Korea \\ ${ }^{4}$ Department of Control \& Instrumentation Engineering, Kangwon National University, Jungangno 1-ga, \\ Samcheok-si, Kangwon-do 245-711, Republic of Korea
}

Received March 3, 2009 and accepted July 27, 2009

\begin{abstract}
The purpose of this study was to observe changes in electromyographic activity appeared in shoulder and scapular muscles when one wears no wrist extension orthosis, short wrist extension orthosis and long wrist extension orthosis when performing two model assembling operations. The study subjects were 16 adults who use right hand and did not have any functional disorder on their wrist or the upper limbs without any orthopedic surgery. The study subjects were asked to do the two model assembling operations without wrist extension orthosis, with a short wrist extension orthosis and with a long wrist extension orthosis. As a result of repetitive assembling operation that requires shoulder movement, electromyographic activity in upper trapezius and serratus anterior increased significantly when the subjects wore the short and long wrist extension orthoses compared to none. When the subjects performed repetitive assembling operation that requires shoulder stability, electromyographic activity in upper trapezius, lower trapezius, serratus anterior, and anterior deltoid showed significant increase when they wore the short and long wrist extension orthoses compared to none. Especially, the upper trapezius showed significantly high electromyographic activity when they wore the long wrist extension orthosis compared to a short one.
\end{abstract}

Key words: Shoulder and scapular muscles, Valpar component work samples, Wrist-extension orthosis

\section{Introduction}

Work-related musculoskeletal disorders (WRMDs), also known as overuse injuries, account for a significant proportion of work injuries. There are several known risk factors for upper-extremity muscle injuries, including repetitive and continuous-flow assembly work ${ }^{1}$. WRMDs are a group of painful disorders of muscles, tendons, and nerves that can be induced by work activities

*To whom correspondence should be addressed

E-mail: minye@yonsei.ac.kr that are frequent and repetitive, or that involve awkward postures ${ }^{2}$.

Carpal tunnel syndrome (CTS) is a common work-related disorder and is a major cause of impairment and disability in the workplace. It is considered a clinical entity, and diagnosis is based on a symptom complex that includes numbness, tingling, and/or burning ${ }^{3)}$. Wrist orthoses can be used to immobilize an unstable joint or fracture, to unload a portion of a joint, to eliminate motion in a particular direction, or to modify the range of motion in one or more directions. As such, it may be useful for diminishing the pain associated with CTS and improving 
physical functioning by slowing its progression ${ }^{3-5)}$.

However, Perez-Balke and Buchholz ${ }^{6}$ theorized that wearing a wrist splint for the treatment of a hand or wrist disorder can increase the risk of shoulder injury. Some studies have indeed demonstrated this negative effect of wearing a wrist splint on shoulder muscle activity ${ }^{7,8)}$. For example, Bulthaup et al. ${ }^{7)}$ found that motor-unit recruitment in the four proximal muscles of the shoulder was significantly greater when wearing an AlignRite wrist splint with a tension strap than when not wearing a splint. This finding has been confirmed by other studies showing that shoulder movements are increased by wrist splinting $^{9-11)}$. For example, King et al. ${ }^{10)}$ reported that wearing a wrist splint increases humeral elevation when performing the Jebsen Hand-Function Test and selected activities of daily living, and Mell et al. ${ }^{11,12)}$ demonstrated that wearing a DonJoy Orthopedics large wrist splint increases the active shoulder elevation when picking an object from a bin. Such increases in shoulder muscle activity and movement could lead to fatigue and increased stress in the shoulder ${ }^{9,11)}$.

However, these aforementioned studies did not consider the rhythmic interaction of the scapular muscles during scapular movement that occurs when the shoulder is repeatedly abducted or flexed. In addition, as the subjects were patients with wrist pain, these studies focused on activities of daily living. The present study therefore assessed the effect of wearing a wrist-extension orthosis (short or long) compared to freehand activity (i.e., no orthosis) on Electromyographic (EMG) activity in the shoulder and scapular muscles when performing two model-assembly operations.

\section{Methods}

\section{Subjects}

A cohort of 16 seated workers who normally worked in a seated position was recruited in Wonju city. The subjects were selected from 12 laboratories by consecutive sampling. The inclusion criteria required that subjects were right-hand dominant, free of dysfunction or pain in the upper limb and hand, and had not undergone orthopedics surgery. Ethical approval was obtained from the Health Sciences Human Ethics Committee of the Yonsei University Faculty, and the subjects provided written informed consent prior to their participation. The characteristics of the subjects are summarized in Table 1 .

\section{Instrumentation}

Wrist extension orthoses

The brace type used in this study can be used to prevent or heal CTS or hand injuries in workers. The particular short and long styles of wrist braces
Table 1. General characteristics of the subjects $(\mathbf{N}=16)$

\begin{tabular}{cc}
\hline Variable & Subjects \\
\hline Age $(\mathrm{yr})$ & $23.1 \pm 2.4$ \\
Height $(\mathrm{cm})$ & $174.6 \pm 3.5$ \\
Mass $(\mathrm{kg})$ & $70.2 \pm 10.5$ \\
Arm length $(\mathrm{cm})$ & $53.4 \pm 2.3$ \\
\hline
\end{tabular}

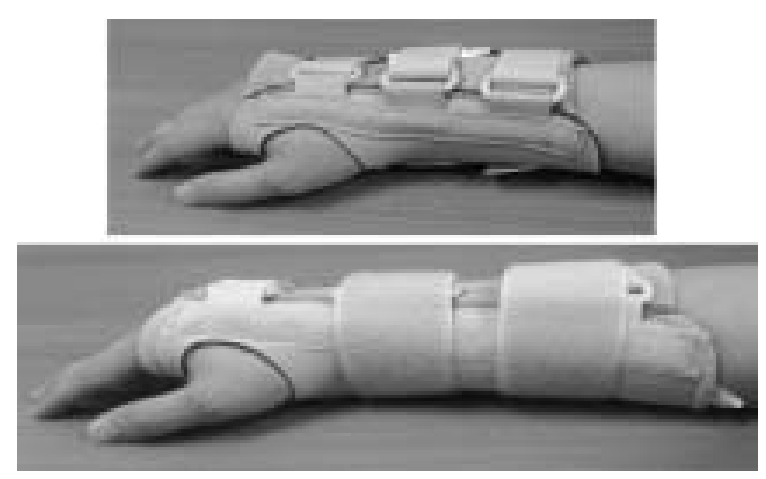

Fig. 1. Themoskin ${ }^{\circledR}$ Wrist Orthosis.

(Thermoskin $^{\mathrm{TM}}$ \& Trioxon $^{\mathrm{TM}}$, Swede-O, North Branch, MN, USA) used (Fig. 1) were chosen because they are frequently prescribed and because there is no other notable difference in their styles apart from their length. The orthoses were fitted to the right hand at a wrist dorsiflexion angle of between $15^{\circ}$ and $30^{\circ}$, which is the recommended range for optimal function ${ }^{7}$.

Valpar component work samples (VALPAR International Corporation, Tucson, Arizona)

The Valpar Component Work Samples (VCWS), which were designed for use in the field of vocational evaluation, were used in the present study. These work samples have become standard-setting vocational evaluation tools and have received the attention of professionals in other health-related disciplines ${ }^{13}$.

Valpar Component Work Sample No.7 (VCWS 7) is a square box $(69.85 \times 69.22 \mathrm{~cm})$ constructed from particle board and weighting $22.23 \mathrm{~kg}$. The evaluee sits in front of the work-sample board and, after a brief practice session, sorts 144 plastic chips into the correct slots. Each chip is identified in one of the following ways: color and letter; color and number; or color, letter, and number. The bottom row of slots constitutes the practice section. VCWS 7 assesses the ability to make rapid sorting decisions involving several levels and combinations of visual discrimination. This work sample simulates light work and involves repetitive finger grasping and reaching that required shoulder movement. The task consisted of rounding up chips from a marked position $5 \mathrm{~cm}$ from the 


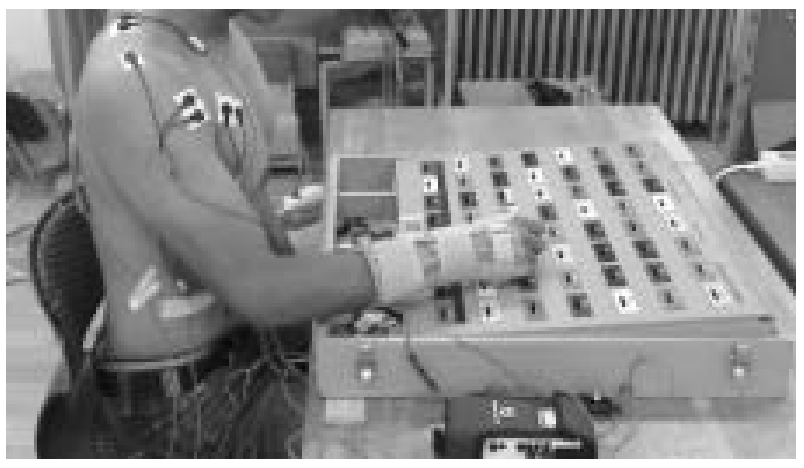

Fig. 2. Repetitive assembling operation (requires shoulder movement).

center of the board and $15 \mathrm{~cm}$ from the front of the table. All processes set up for the handswitch were checked at the beginning and ending. The subject pushed the handswitch following a verbal instruction such as "touch", and sorted the 20 chips into holes when given a verbal instruction such as "start". The handswitch was pushed at the end of the task. An adjustable-height table and a chair with no backrest were used to set the initial sitting posture so as to ensure that the hips and knees were flexed by $90^{\circ}$ (Fig. 2).

Valpar Component Work Sample No.204 (VCWS 204) is a square box $(43.18 \times 43.18 \mathrm{~cm})$ constructed from particleboard and weighing $9.07 \mathrm{~kg}$. The flat surface has 20 holes, each of which contains a threaded jack pin. A nylon string is attached to a metal plug to the left of the 20 holes when viewing the box from the front. VCWS 204 simulates sedentary work requiring small objects to be turned with both right and left hands, and using tweezers to grasp, lift, and hold small items with one hand while performing other operations with the other hand; in this case, threading a nylon string through small holes in the jack pins. The nylon string was picked up with the left hand, and then the pincet was picked up with the right hand. The subject pushed the handswitch following a verbal instruction such as "touch", and picked up 10 jack pins when given a verbal instruction such as "start". The handswitch was pushed at the end of the task. The boundaries of the task areas were marked by tape on the table so as to minimize errors (Fig. 3).

\section{Surface EMG recording}

We collected and amplified EMG data from the anterior deltoid, upper trapezius, pectoralis major, lower trapezius, and serratus anterior muscles (Noraxon TeleMyo 2400T), and analyzed the obtained data (MyoResearch Master Edition 1.06 XP, Noraxon, Scottsdale, AZ, USA). The disposable Ag-AgCl surface electrodes were positioned at an interelectrode distance of

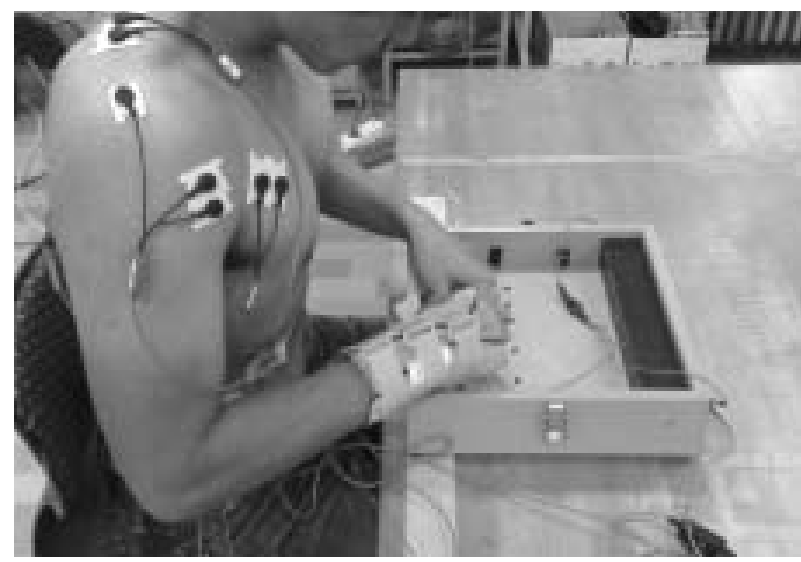

Fig. 3. Repetitive assembling operation (requires shoulder stability).

$2 \mathrm{~cm}$ (Bule Sensor, Medicotest, Olstykke, Denmark).

Surface electrodes attached to the skin after it had been shaved and then cleaned using alcohol detected the muscle activities on the subject's right side. The electrode locations were as follows: (1) upper trapezius - slightly lateral to and halfway between the cervical spine at C-7 and the acromion; (2) lower trapezius-approximately 5 $\mathrm{cm}$ down from the scapular spine; (3) serratus anterior just below the axillary area, at the level of the inferior tip of the scapula and with the electrodes anterior to the latissimus dorsi muscle (4) anterior deltoid - anterior aspect of the arm, approximately $4 \mathrm{~cm}$ below the clavicle; and (5) pectoralis major - the chest wall at an oblique angle toward the clavicle, approximately $2 \mathrm{~cm}$ below the clavicle, just medial to the axillary fold ${ }^{14)}$.

The raw signal was full-wave rectified and digitally filtered (Lancosh FIR) between $20 \mathrm{~Hz}$ and $450 \mathrm{~Hz}$, and sampled at 1,000 Hz. The EMG data were processed into root-mean-square (RMS) values in a window comprising $300 \mathrm{~ms}$ of data. Average root mean squared EMG (rmsEMG) values were calculated from three timed recordings made in each orthosis condition (i.e., long, short, and none). All subjects performed reference voluntary contractions (RVCs) of the five shoulder muscles (anterior deltoid, pectoralis major, serratus anterior, upper trapezius, and lower trapezius); the experimental data could then be normalized to the submaximal RVCs, thus minimizing the effects of interindividual differences. RVCs of the anterior deltoid, pectoralis major, and serratus anterior muscles were obtained whilst holding a 0.5 $\mathrm{kg}$ dumbbell in each hand, with the arm flexed at 90 in the frontal plane and parallel to the floor. RVCs of the upper trapezius and lower trapezius shoulder muscles were obtained whilst holding a $0.5-\mathrm{kg}$ dumbbell in each hand, with the arm abducted at 90 in the frontal plane and parallel to the floor. The measurements were made 
with the subjects sitting with their hips and knees each at an angle of 90. Subjects completed five 15-s submaximal exertions with a 5-min rest period between contractions. Normalization of the data was achieved using MyoResearch 1.06 software, and data are expressed as mean percentages relative to RVCs (i.e., $\% \mathrm{RVC}=$ Average $\mathrm{rmsEMG} / \mathrm{RVC} \times 100$ ).

\section{Experimental conditions}

The applied task and orthosis condition were selected in a randomized order. The work environment was set up at a distance from the trunk corresponding to $70 \%$ of the arm length in order to prevent excessive trunk motion ${ }^{15)}$. All subjects performed the tasks unsplinted and when wearing long and short wrist-extension orthoses. The same configuration was maintained for the duration of the work period. A 5-min rest period was permitted between multiple repetitions under the different orthosis conditions. A longer rest period was permitted between task conditions to prevent muscle fatigue. In addition, the subjects used a metronome during the tasks to allow them to perform the task at a constant frequency, over a 1-h period. The tasks involved sorting 20 chips into holes within a 60 -s period and picking up 10 jack pins within a 120 -s period, and were based on the average time taken to complete the tasks during training (Figs. 2, 3).

\section{Statistical analysis}

The SPSS statistical package (SPSS 12.0, Chicago, IL, USA) was used to analyze differences in the shoulder and scapular muscle activities. The Kolmogorov-Smirnov test was used to test for a normal distribution prior to using parametric statistics. The significance of differences between when working freehand and with a long orthosis and with a short orthosis were tested by one-way repeated-measures ANOVA, with significance defined as being present when $p<0.05$. For the significant main effect, Bonferroni's correction was performed to identify the specific mean differences.

\section{Results}

Electromyography data: required shoulder movement

One-way repeated-measures ANOVA tests of normalized EMG data obtained during repetitive assembling operation that requires shoulder movement revealed significant differences in the upper trapezius $(p<0.001)$ and serratus anterior $(p<0.01)$ muscles between the freehand, short-orthosis, and long-orthosis conditions. In multiple comparisons, the activities of the upper trapezius and serratus anterior muscles were significantly higher in the short- and long-orthosis conditions than in the freehand condition (Bonferroni-corrected: upper trapezius, $p<0.001$ and $p<0.001$, respectively; and serratus anterior, $p<0.01$ and $p<0.01$, respectively), but they did not differ significantly between the short- and long-orthosis conditions. The activities of the lower trapezius, anterior deltoid, and pectoralis major were unaffected by the orthosis condition (Fig. 4, Table 2).

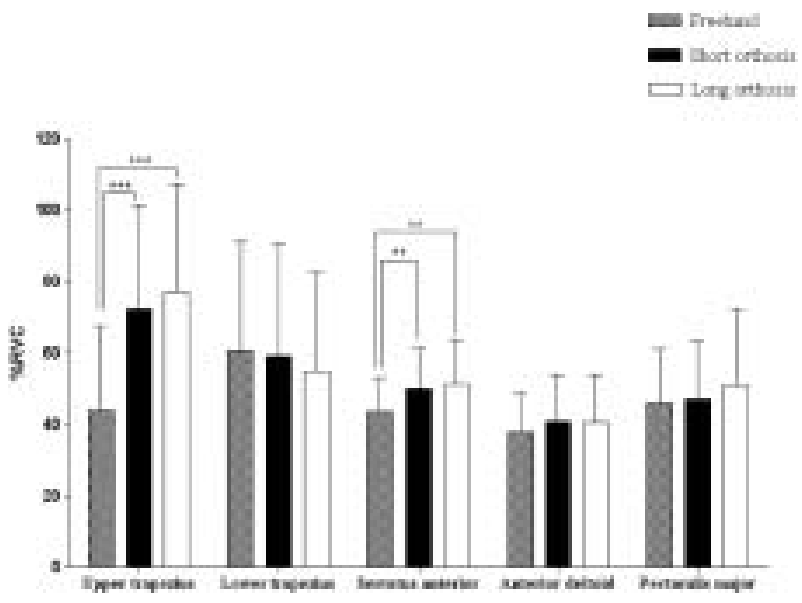

Fig. 4. Muscle activation in the three orthosis conditions, all of which require shoulder movement: none, short, and long. ${ }^{*} p<0.05$ (Bonferroni-corrected), $* * p<0.01, * * * p<0.001$.

Table 2. Normalized EMG data of the shoulder and scapular muscles in the three study conditions during repetitive assembling operation (requires shoulder movement)

\begin{tabular}{ccccc}
\hline \multirow{2}{*}{$\begin{array}{c}\text { Muscle activities } \\
(\% R V C)\end{array}$} & \multicolumn{3}{c}{ Wrist Orthosis Conditions } & \multirow{2}{*}{$p$} \\
\cline { 2 - 4 } & Freehand & Short orthosis & Long orthosis & \\
\hline Upper trapezius & $43.81 \pm 26.11^{*}$ & $72.24 \pm 32.03$ & $77.26 \pm 33.73$ & 0.000 \\
Lower trapezius & $60.81 \pm 33.05$ & $59.14 \pm 33.82$ & $54.82 \pm 29.72$ & 0.485 \\
Serratus anterior & $43.76 \pm 12.71$ & $50.10 \pm 13.77$ & $51.25 \pm 13.80$ & 0.001 \\
Anterior deltoid & $37.79 \pm 16.17$ & $40.76 \pm 17.19$ & $40.59 \pm 17.49$ & 0.084 \\
Pectoralis major & $45.98 \pm 17.48$ & $47.07 \pm 19.38$ & $50.93 \pm 21.72$ & 0.213 \\
\hline
\end{tabular}

*mean $\pm \mathrm{SD}$. 
Electromyography data: required shoulder stability

One-way repeated-measures ANOVA tests of normalized EMG data obtained during repetitive assembling operation that requires shoulder stability revealed significant differences in the upper trapezius $(p<0.01)$, lower trapezius $(p<0.01)$, serratus anterior $(p<0.001)$, and anterior deltoid $(p<0.001)$ muscles between the freehand, short-orthosis, and long-orthosis conditions. In multiple comparisons, the activities of the upper trapezius, lower trapezius, serratus anterior, and anterior deltoid muscles of the proximal joints were significantly higher in the short- and long-orthosis conditions than in the freehand condition (Bonferroni-corrected: upper trapezius, $p<0.01$ and $p<0.01$, respectively; lower trapezius, $p<0.01$ and $p<0.01$, respectively; serratus anterior, $p<0.001$ and $p<0.001$, respectively; and anterior deltoid, $p<0.001$ and $p<0.01$, respectively). Upper-trapezius muscle activity was significantly greater in the long-orthosis condition than in the short-orthosis condition (Bonferroni-corrected $p<0.05)$. The activity of the pectoralis major was unaffected by the orthosis condition. (Fig. 5, Table 3).

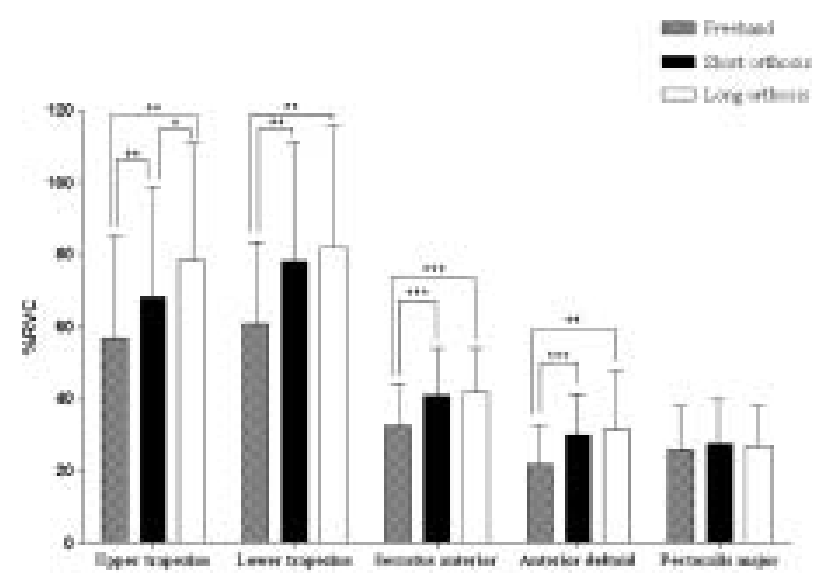

Fig. 5. Muscle activation in the three orthosis conditions, all of which require shoulder stability: none, short, and long. ${ }^{*} p<0.05$ (Bonferroni-corrected), $* * p<0.01, * * * p<0.001$.

\section{Discussion}

The purposes of this study were to compare the effects of wearing the wrist-extension orthosis to freehand, and to establish any effect of the length of the orthosis (short or long) on shoulder muscle activities when subjects performed two simulated assembly tasks that are representative tasks of those used in the manufacturing industry. The activities of the following muscles were assessed: (1) the anterior deltoid and pectoralis major muscles, which are commonly used to move the humerus; (2) the upper trapezius, lower trapezius, and serratus anterior muscles, which are used in combination to produce a coupled-force scapular upward rotation during scapulohumeral motion ${ }^{15)}$. These muscles have also been selected for study by many researchers in surface EMG studies of upper-extremity musculoskeletal disorders ${ }^{16-20)}$.

One of the tasks selected in our study involved picking up small chips and putting them into grooves, requiring shoulder movements, similar to a simple task performed often in bank and post-office work. Especially, this task was associated with a significant increase in the muscle activation of the upper trapezius and serratus anterior muscles, which in combination produce a coupling force to move the scapula toward upward rotation. Wearing a wrist orthosis during the task allowed a more compensated activation of the shoulder musculature relative to not wearing a splint ${ }^{11)}$.

The second task involved holding up jack pins through which nylon strings were inserted, requiring the maintenance of a shoulder stability position, similar to one of the precision tasks required during clock assembly work. When subjects performed this task there was an increase in the activation of the anterior deltoid muscles, the function of which is shoulder abduction, and the upper trapezius, lower trapezius, and serratus anterior muscles, which operate in combination to produce a coupled force to move the scapula toward upward rotation. Our results clearly show that this task produces a large shoulder and scapular motion ${ }^{16,21)}$. This may be explained by the

Table 3. Normalized EMG data of the shoulder and scapular muscles in the three study conditions during repetitive assembling operation (requires shoulder stability)

\begin{tabular}{ccccc}
\hline \multirow{2}{*}{$\begin{array}{c}\text { Muscle activities } \\
(\% \mathrm{RVC})\end{array}$} & \multicolumn{3}{c}{ Wrist Orthosis Conditions } & \multirow{2}{*}{$p$} \\
\cline { 2 - 4 } & Freehand & Short orthosis & Long orthosis & \\
\hline Upper trapezius & $56.79 \pm 28.13^{*}$ & $68.23 \pm 32.11$ & $78.60 \pm 35.22$ & 0.002 \\
Lower trapezius & $60.86 \pm 21.66$ & $78.04 \pm 33.80$ & $82.26 \pm 38.23$ & 0.009 \\
Serratus anterior & $32.41 \pm 13.77$ & $41.06 \pm 15.00$ & $41.92 \pm 14.18$ & 0.000 \\
Anterior deltoid & $21.81 \pm 12.56$ & $29.93 \pm 16.62$ & $31.58 \pm 19.19$ & 0.000 \\
Pectoralis major & $25.98 \pm 12.88$ & $27.71 \pm 13.79$ & $27.11 \pm 12.68$ & 0.252 \\
\hline
\end{tabular}

*mean $\pm \mathrm{SD}$. 
increased muscle activities in the shoulder and scapular muscles $^{22)}$. In particular, there was too much compensative shoulder movement-in this case shoulder abduction-when subjects worked while wearing the wristextension orthosis, and thus they were at greater risk of resultant shoulder problems than when working unsplint$\mathrm{ed}^{11,23,24)}$

In the present study we selected a task that required shoulder movement and a task that required maintenance of a shoulder stability position, and these resulted in slightly different patterns of response. Our results demonstrate an increase in compensative shoulder movement that requires more wrist movement than shoulder movement. The results are consistent with those of previous studies $^{22,25}$. Our study also complements the finding that the character of the task determines the muscles that are affected. Although less arm movement was required during the shoulder stability task, we found an increase in compensative shoulder movement when the subject wore a wrist-extension orthosis.

Bulthaup et al. ${ }^{7)}$ found no significant difference in muscle activity with orthoses of different lengths. However, in the present study, a significant difference was noted between shoulder muscle activities in subjects wearing short and long wrist-extension orthoses. When the subjects performed a repetitive assembly operation that required maintenance of a shoulder stability position, the upper trapezius muscle exhibited significantly higher electromyographic activity when the subjects wore the long wrist-extension orthosis compared to the short one. The upper trapezius muscle has been closely associated with shoulder muscle pain in studies of musculoskeletal disease in the upper extremity ${ }^{18)}$. If used continuously, this muscle would result in myofascial pain syndrome in the shoulder. Therefore, the appropriate selection of wristextension orthosis is very important in the prevention of disorders of the shoulder muscles resulting from overuse $^{23,24)}$.

Levin et al. ${ }^{24)}$ demonstrated that the repeatedly abnormal cocontraction of the proximal joint induces exposure to secondary risk factors. Unfortunately, many of the jobassistance tools that are available are associated with these problems, such as the putative effect of wrist orthoses on the shoulder musculature ${ }^{12,26-28)}$. In the present study, although the subjects practiced the specified movement while wearing the orthoses, it is necessary to explore in real time the effects of working for a long time while wearing an orthotic, under conditions whereby subjects might have developed an overuse syndrome. It has been shown that cumulative trauma diseases lead to musculoskeletal disease. Although wearing a wrist-extension orthosis is an essential therapeutic measure, patients need to be educated as to its appropriate use to prevent sec- ondary disease. This information will help therapists to provide more specific guidelines for maximizing the many positive effects and minimizing the potentially negative effects of wrist orthoses on a range of motion requirements and quality of movements.

This study had several limitations. First, we used healthy subjects rather than patients with a hand injury. None of the subjects had any experience of a wearing wrist-extension orthosis, so they had to be trained for many hours. However, we were unable to show compensatory shoulder movement associated with wearing the wrist orthosis for a long time. Second, all of the subjects were men. Women did not wish to participate in this study because they had to remove some of their clothes. Third, we did not analyze the onset times of shoulder and scapular muscle activity because we were not analyzing motion in three dimensions.

Future studies should analyze synchronously obtained motion-analysis and EMG data for patterns of longer-term changes and the effects of wrist orthoses with different characteristics on the shoulder and scapular musculature.

\section{Conclusion}

This study compared the EMG activities of shoulder and scapular muscles - which can induce shoulder pain when performing assembly tasks - in freehand, shortorthosis, and long-orthosis conditions. Our results demonstrated that muscle activity increases during a task that required shoulder movement and shoulder stability. Especially, we consider that the length of time spent wearing long wrist extension orthosis should be minimized when performing work that requires maintenance of a shoulder stability position. The appropriate selection of wrist-extension orthosis is very important in the prevention of disorders of the shoulder muscles resulting from overuse.

\section{References}

1) Szeto GP, Straker LM, O'Sullivan PB (2005) EMG median frequency changes in the neck-shoulder stabilizers of symptomatic office workers when challenged by different physical stressor. J Electromyogr Kinesiol 15, 544-55.

2) Punnett L, Wegman DH (2004) Work-related musculoskeletal disorders: the epidemiologic evidence and the debate. J Electromyogr Kinesiol 14, 13-23.

3) Gravlee JR, Vandurme DJ (2007) Braces and splints for musculoskeletal conditions. Am Fam Phys 75, 342-8.

4) Taylor E, Hanna J, Belcher HJCR (2003) Splinting of the hand and wrist. Cur Orth 17, 465-74.

5) Weiss ND, Gordon L, Bloom T, So Y, Rempel DM (1995) Position of the wrist associated with the lowest 
carpal-tunnel pressure: implications for splint design. J Bone Joint Surg 77, 1695-9.

6) Perez-Balke G, Buchholz B (1994) Role of wrist immobilization in the work environment: ergonomics and carpal tunnel syndrome. Work 4, 187-94.

7) Bulthqup S, Cipriani DI, Thomas JJ (1999) An electromyography study of wrist extension orthoses and upper extremity function. Am J Occup Ther 53, 434-40.

8) Chan W, Chapparo C (1999) Effect of wrist immobilization on upper limb function of elderly males. Technol Disabil 11, 39-49.

9) Callinan N (1999) Clinical interpretation of an electromyography study of wrist extension orthoses and upper extremity function. Am J Occup Ther 25, 441-4.

10) King S, Thomas J, Rice MS (2003) The immediate and short-term effects of a wrist extension orthosis on upper extremity kinematics and range of shoulder motion. Am J Occup Ther 57, 517-24.

11) Mell AG, Childress BL, Hughes RE (2005) The effect of wearing a wrist splint on shoulder kinematics during object manipulation. Arch Phys Med Rehabil 86, 1661-4.

12) Mell AG, Freidman MA, Hughes RE, Carpenter JE (2006) Shoulder muscle activity increases with wrist splint with wrist splint use during a simulate upper extremity work task. Am J Occup Ther 60, 320-6.

13) Christopherson B, Hayes $P$ (2006) Vaplar component work samples uses in allied health. Valpar International Corporation, Tucson.

14) Cram JR, Kasman GS, Holtz J (1998) Introduction to surface electromyography. Aspen Publishers, New York.

15) Levin MF, Cirstea CM, Archambault P, Son F, RobyBrami A (2001) Impairment and compensation of reaching in hemiparetic and cerebral palsied patients. In: Bernstein's Traditions in Motor Control, Latash M and Rothwell J (Eds.), Human Kinetics, Idaho.

16) Holte KA, Westgaard RH (2002) Daytime trapezius muscle activity and shoulder neck pain of service workers with work stress and low biomechanical exposure. J Electromyogr Kinesiol 9, 317-25.

17) Neumann DA (2002) Kinesiology of the musculoskele- tal system: foundation for physical rehabilitation. Mosby, St. Louis.

18) Jensen C, Finsen L, Hansen K, Christensen H (1999) Upper trapezius muscle activity patterns during repetitive manual material handling and work with a computer mouse. J Electromyogr Kinesiol 9, 317-25.

19) Sporrong H, Palmerud G, Kadefors R, Herberts P (1998) The effect of light manual precision work on shoulder muscles an EMG analysis. J Electromyogr Kinesiol 8, 177-84.

20) Yoo WG, Yi CH, Kim MH (2007) Effect of a ball-backrest chair on the muscles associated with upper crossed syndrome when working at a VDT. Work 29, 239-44.

21) Decker MJ, Hintermeister RA, Faber KL, Hawkins RJ (1999) Serratus anterior muscle activity during selected rehabilitation exercise. Am J Sports Med 27, 784-91.

22) Bernard BP (1997) Musculoskeletal disorders and workplace factors: a critical review of epidemiologic evidence for work-related musculoskeletal disorders of the neck, upper extremity, and low back. National Institute for Occupational Safety and Health, Cincinnati.

23) Punnett L, Fine LJ, Keyserling WM, Herrin GD, Chaffin DB (2000) Shoulder disorders and postural stress in automobile assembly work. Scand J Work Environ Health 26, 283-91.

24) Adams ML, Franklin GM, Barnhart S (1994) Outcome of carpal tunnel surgery in washington state workers' compensation. Am J Ind Med 25, 527-36.

25) Burtner PA, Anderson JB, Marcum ML, Poole JL, Qualls C, Picchiarini MS (2003) A comparison of static and dynamic wrist splints using electromyography in individuals with rheumatoid arthritis. J Hand Ther 16, 320-5.

26) Cristea CM, Levin MF (2000) Compensatory strategies for reaching in stroke. Exp Brain Res 123, 940-53.

27) Kaminski TR, Bock C, Gentile AM (1995) The coordination between trunk and arm motion during pointing movements. Exp Brain Res 106, 457-66.

28) Das B, Sengupta AK (1995) Computer-aided human modeling programs for workstation design. Ergonomics 38, 1958-72. 